\title{
Efficient Cluster Formation Protocol in WSN
}

\author{
${ }^{1}$ Mr. Dharmesh Dhabliya, ${ }^{2} \mathrm{Mr}$. Rahul Sharma \\ ${ }^{1}$ Research Scholar, Vishwakarma Institute of Information Technology Pune India \\ ${ }^{2}$ Research Department, Auricle Technologies Bikaner India \\ dharmeshdhabliya@gmail.com
}

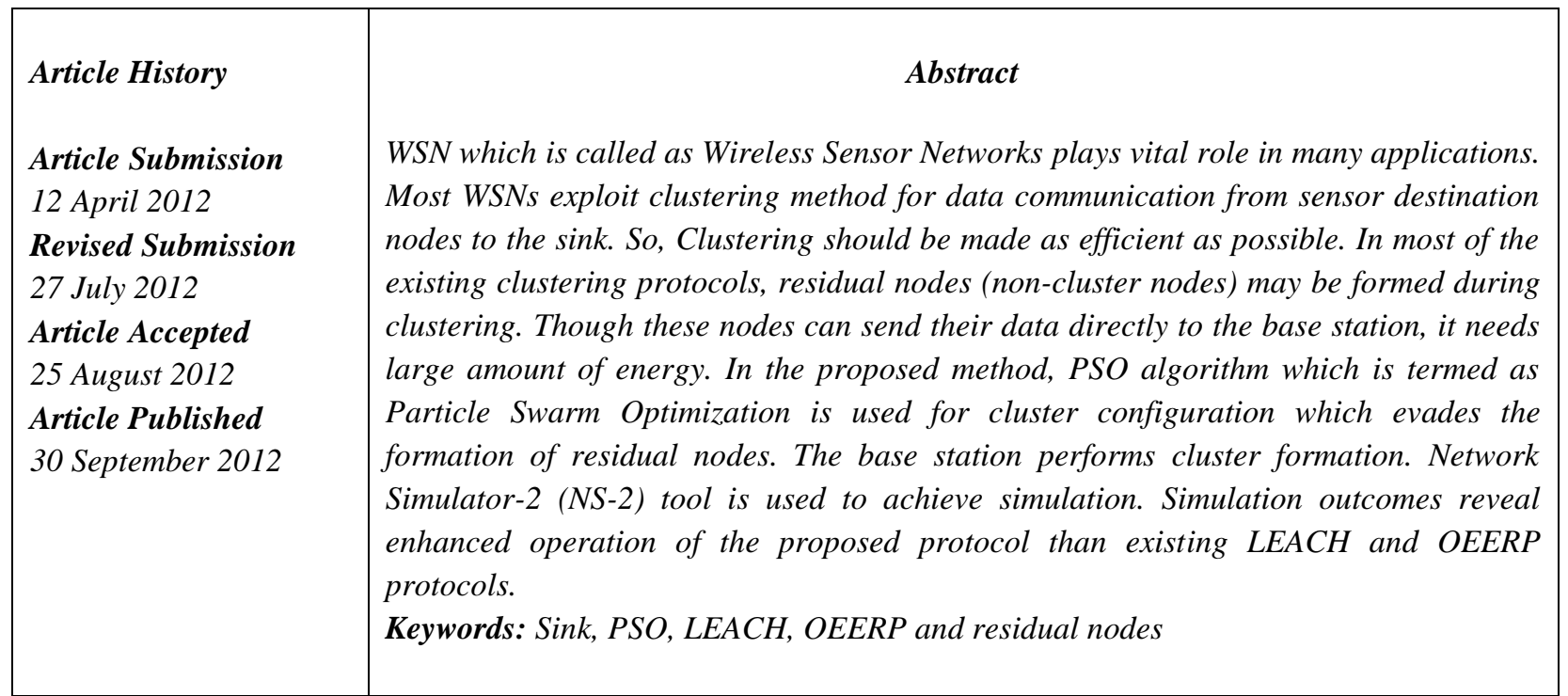

\section{Introduction}

Wireless Sensor Network (WSN) is network which acts wireless with various set of sensor nodes, deployed in an environment to sense the required attribute that includes physical quantities such as pressure and temperature. A sensor node includes sensor for sensing, microcontroller for processing, Analog to Digital Converter, Transceiver, Power Source and some additional gadgets [1]. The sensor nodes can sense the data and transmit to destination nodes. WSN has many emerging applications in various fields including Military tracking and monitoring to track enemies/security detection, Environmental monitoring, Habitat Monitoring, Health Monitoring and also in Agricultural and Industrial applications [2]. In WSN, in order to transmit data from the sensor node to the destination, various methods are deployed that includes Single \& multi hop model, cluster Model, tree model and chain model.

Most of the sensor networks use this clustering method for data transmission. Various algorithms are available for formation of clusters including LEACH and OEERP. LEACH is a self-organizing, adaptive bunch protocol which contains the cluster head with high energy [3]. OEERP is a cluster based protocol where Cluster-Heads change for every time-slot [4].

In both of these clustering protocols, all sensor nodes cannot always be part of a cluster during the cluster formation process. There may be cases where some nodes are left out of the cluster because of being not within the transmission range of the cluster-head and they are called as non-cluster nodes or residual nodes. If there is any sensed data in these non-cluster nodes, it might loss. In the proposed Efficient Cluster Formation Protocol (ECFP) protocol, PSO algorithm is employed for cluster formation which prevents the residual nodes formation.

ISSN: 2250-0839 


\section{Related Works}

Most research proposals in clustering area based WSNs have concentrated on the efficient energy utilization, effective data gathering, aggregation mechanisms and network lifetime. In this section, some of the cluster based protocols are reviewed.

\section{LEACH Protocol}

LEACH is a self-organized, cluster oriented protocol that elects Cluster-Heads based on a certain probability. Once they are elected, each sensor node determines its $\mathrm{CH}$ by identifying the least communication energy requirement. It also minimizes the energy consumption by turning off the radio components except at the transmit time. On receiving data from every nodes, CHs perform aggregation on data and transmits to the sink. It involves the following rounds:

\section{a. Advertisement Phase}

Cluster-Heads advertise its election by broadcasting a advertise message to each nodes using CSMA MAC protocol. The election of $\mathrm{CH}$ is done in the following manner. The threshold value $\mathrm{T}$ which is given below.

$$
T= \begin{cases}\frac{S}{1-S *\left(R_{C} \bmod \frac{1}{S}\right)} & \text { if } N \in N_{C} \\ 0 & \text { otherwise }\end{cases}
$$

\section{b. Cluster Set-Up Phase}

After the decision made by nodes to join the cluster, the respective $\mathrm{CH}$ should be informed which is a member of the cluster. This vital information can be transmitted to the head cluster using the CSMA MAC protocol. Based on this information, clusters are formed.

\section{c. Steady-State Phase}

After the formation of clusters, CHs create a TDMA schedule which provides all nodes the transmission time. After the reception of data from cluster members, data aggregation is performed and finally aggregated data to be transmitted to the sink. Thus LEACH provides rotation of high-energy Cluster-Heads. [3].

\section{Improved LEACH Protocol}

This provides modifications to the LEACH protocol that each cluster contains Head of cluster $(\mathrm{CH})$, sub-CH and Sensor nodes. Normally, $\mathrm{CHs}$ receive data from nodes, perform data aggregation and transmits to the base station. So, continuous reduction of energy level takes place resulting in the earlier $\mathrm{CH}$ death. The availability of the sub-SH is essential when $\mathrm{CH}$ dies. The sub- $\mathrm{CH}$ node acts as $\mathrm{CH}$ only in the absence of the $\mathrm{CH}$ [5].

\section{OEERP Protocol}

It is a hierarchical routing protocol which minimizes data loss in individual nodes and provides uniform energy drain of all sensor nodes.

\section{a. Cluster Formation Phase}

This phase initiates with the arbitrary selection of Cluster-Head which process within certain time known as time slot. After selection of $\mathrm{CHs}$, they advertise all nodes in the scenario about its selection by broadcasting a packet. Nodes that are in range of the Cluster-Heads, receive the packet and sends request to join the cluster. In the next 
time-slot, arbitrary selection process of $\mathrm{CHs}$ take place thereby forming new clusters.

\section{b. Data Gathering Phase}

After Cluster Formation phase, data gathering takes place in such a way that all cluster nodes transmit their data to their respective Cluster-Head. If there is any sensed data resides in individual nodes, it transmits to the nearest cluster-node which transmits the same to its $\mathrm{CH}$.

\section{c. Data Aggregation Phase}

Data Gathering should be followed by performing data aggregation process. Data Aggregation is done by the CH on data collected from various nodes. Data aggregation is carried out to avoid redundant data transmission, thereby providing summarized data by performing any of the aggregation functions.

\section{d. Data Transmission Phase}

In this final phase, the aggregated data from the Cluster-Head will be transmitted to the BS based upon the allotted time interval. The interval is allotted using Time Division Multiple Access scheme [4].

\section{Proposed Work}

The proposed ECFP protocol is an efficient clustering protocol which performs clustering in a well-organized manner. The base station performs clustering process. It exploits Particle Swarm Optimization (PSO) algorithm to accomplish clustering. The PSO initiates with the random deployment of sensor nodes called particles in the field. The base station collects information of all nodes by broadcasting "Info_Collection" message. All nodes reply the message with its Location, Velocity and Energy information to the base station. The base station remains until it receives "Info Reply" from all sensor nodes, stores and then performs clustering.

The various steps in the proposed protocol involve:

a. Cluster Establishment Phase

b. Cluster Assistant Selection Phase

c. Cluster Head Election Phase

d. Data Accumulation and Transmission Phase

\section{a. Cluster Establishment Phase}

The steps in Particle Swarm Optimization (PSO) algorithm include the following:

- Determination of current location and velocity of each particle

- Computation of Fitness values for all particles

- Determination of Local Best value

- Updation of Velocity and Location

- Repetition of steps 2 and 3

- Determination of Global Best value

- Cluster formation with Global Best as Clustering particle 


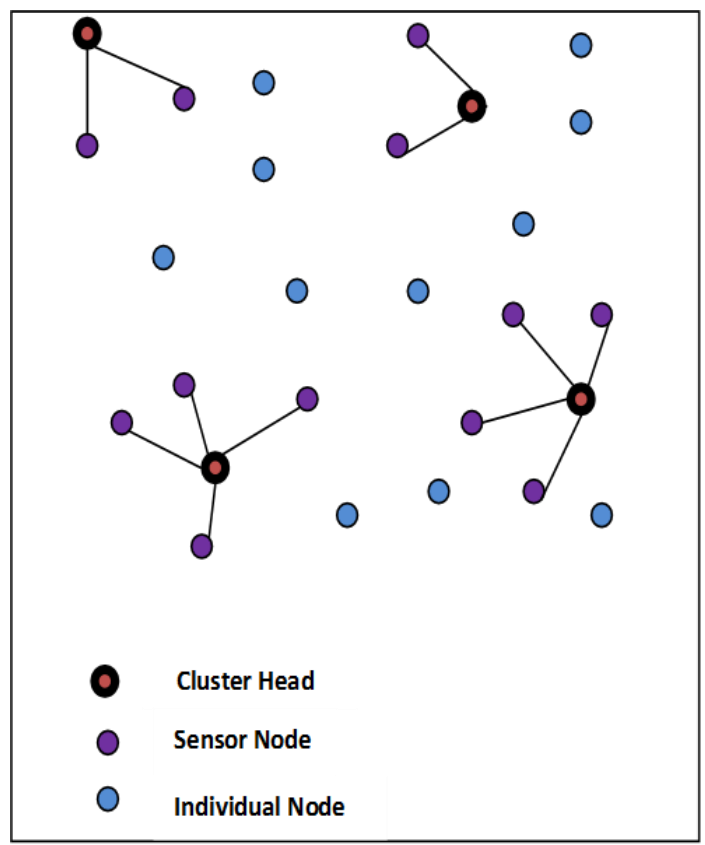

Fig. 1. Cluster Formation at time-slot 1

Initially, all particles reply for the Info Collection message to the base station with the following information:

- Position $\mathrm{X}_{\mathrm{i}}$ with parameters $(\mathrm{x}, \mathrm{y})$

- Velocity $\mathrm{V}_{\mathrm{i}}$ with parameters $\left(\mathrm{v}_{1}, \mathrm{v}_{2}\right)$

- Energy of the particle

After receiving position and velocity information from all particles, fitness value is calculated for all particles. Computation of Fitness value requires the consideration of cluster radius for each particle and number of sensors to be covered within the radius.

Fitness value can be computed using the formula:

$$
F=\alpha_{1} x_{1}+\alpha_{2} x_{2}+\left(1-\alpha_{1}-\alpha_{2}\right) x_{3}
$$

Where,

F-Fitness value

$\alpha_{1}, \alpha_{2}-$ constants between 0 and 1

$$
x_{1} \quad=\quad \frac{\operatorname{Avg} E_{C N}}{E_{p}}
$$

After computing Fitness values, Local Best value is selected based upon the maximum fitness-value for the current iteration. The Location and Velocity of each particle is updated for next iteration based on the following update equation:

$$
V_{\text {Update }}=\omega . V_{i-1}+\omega_{1}\left(X_{i-1}-X_{i}\right)+\left(X_{i-1} . X_{i}\right)
$$


Where,

$V_{\text {Update }}$-Updated Velocity

$\omega$-weight of node location

$\omega_{1,} \omega_{2}$-weights of node mobility

$\mathrm{V}_{\mathrm{i}-1}$-Last velocity

$X_{i-1}$-Last location

$X_{i}$-Current location

Hence

$$
X_{\text {Update }}=X_{i-1}+V_{\text {Update }}
$$

Local Best value is calculated for every iteration i.e., the maximum fitness value in each iteration. The maximum fitness value among all iterations is known as Global Best value and the particle is Global Best particle.

With the Global Best particle as the Clustering Particle, clustering will be carried out. Cluster radius is considered for every cluster with Clustering particle as the center point. Those particles covered within the cluster radius of a particular Clustering particle join up to form a cluster.

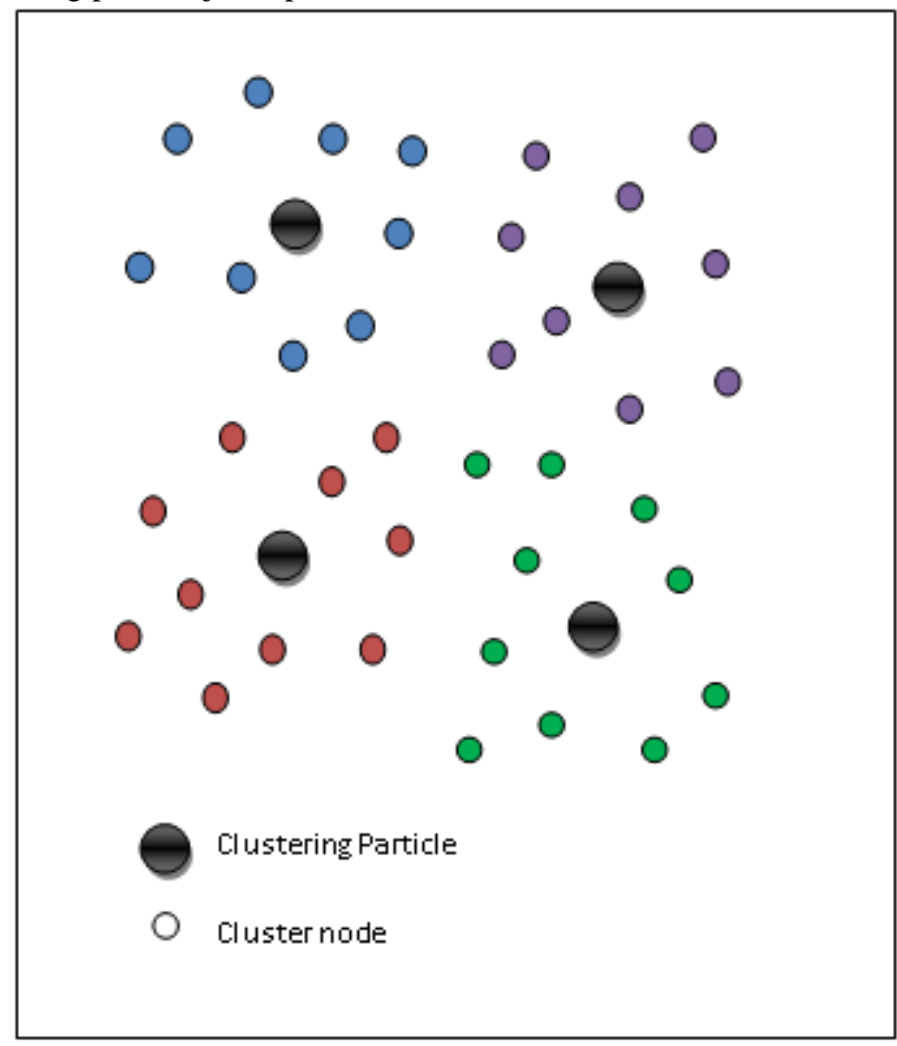

Fig. 2. Cluster Formation with Clustering Particle

\section{b. Cluster Assistant Selection Phase}

Cluster Formation is followed by Cluster Assistant (CA) selection. Cluster Assistant assists Cluster Head node. It downgrades the work of Cluster-Head. Among the newly formed clusters, particle with the maximum fitness value i.e., the optimized particle in each cluster is selected as the Cluster Assistant (CA) node.CA node maintains information in its cluster. It transmits cluster information and CA information to every nodes in the network. CA in local clusters identifies Cluster-Head in each round. It broadcasts Cluster-Announcement message to all nodes with information about Cluster ID, CA ID and Cluster list. Cluster Assistant node performs the following: 
- It performs Cluster-Head election for each round in a distributed manner.

- It provides energy updating of sensor nodes in the cluster for every round.

- It does not perform sensing.

CA is not allowed to perform sensing in order to prevent the earlier death of the node.

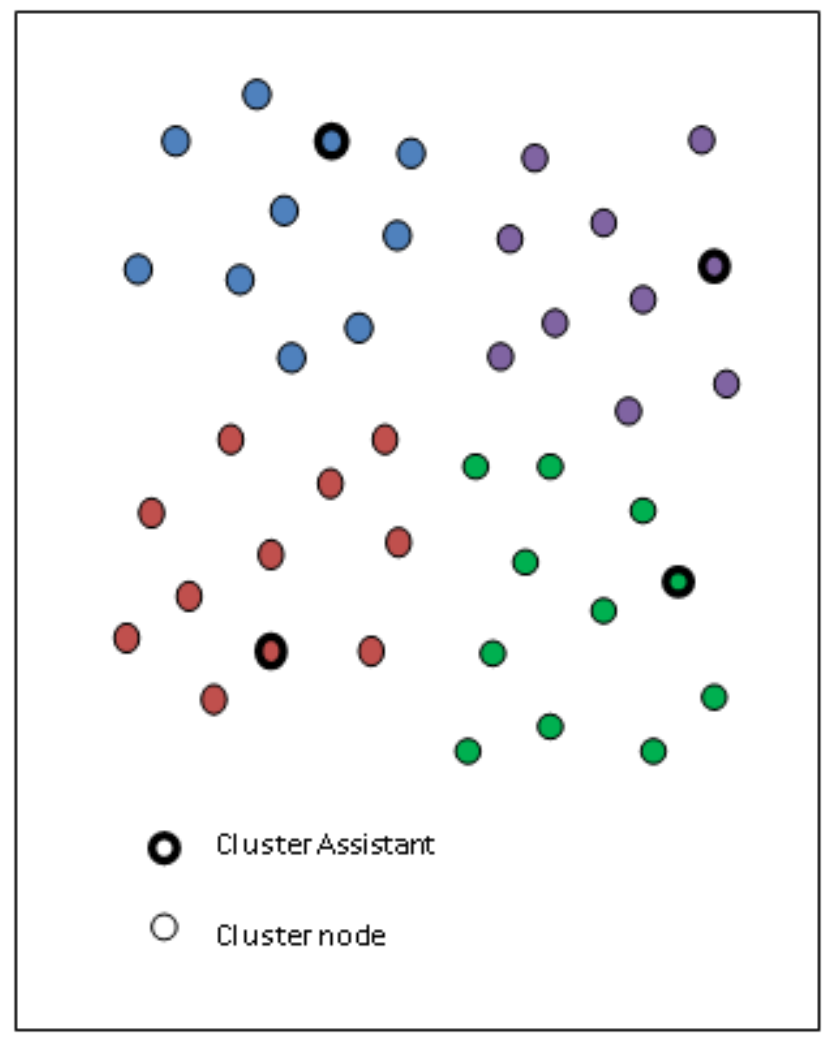

Fig. 3. Clusters with Cluster Assistant node

\section{c. Cluster Head Election Phase}

This Cluster Head $(\mathrm{CH})$ determination is achieved by Cluster Assistant node in the cluster. It exploits Particle Swarm Optimization (PSO) algorithm for $\mathrm{CH}$ election. CA follows the same procedure as in cluster establishment phase. After Cluster-Head selection, it broadcasts "Head Announcement" to each nodes in the network. All cluster nodes records the ID of their respective Cluster Head. Normally, energy loss of CH is higher than cluster nodes. CA, which updates the energy of all nodes, performs again head cluster election for the next round.

\section{d. Data Accumulation and Transmission Phase}

Following Cluster Assistant and $\mathrm{CH}$ election, data gathering is performed. Each sensor oriented node in the cluster transmits its data to $\mathrm{CH}$ of its cluster. After receiving data from sensor nodes, data aggregation is performed. Data aggregation is performed to prevent data loss and to achieve compression of data for minimized energy consumption. The aggregated data will be transmitted to the sink by the $\mathrm{CH}$. 


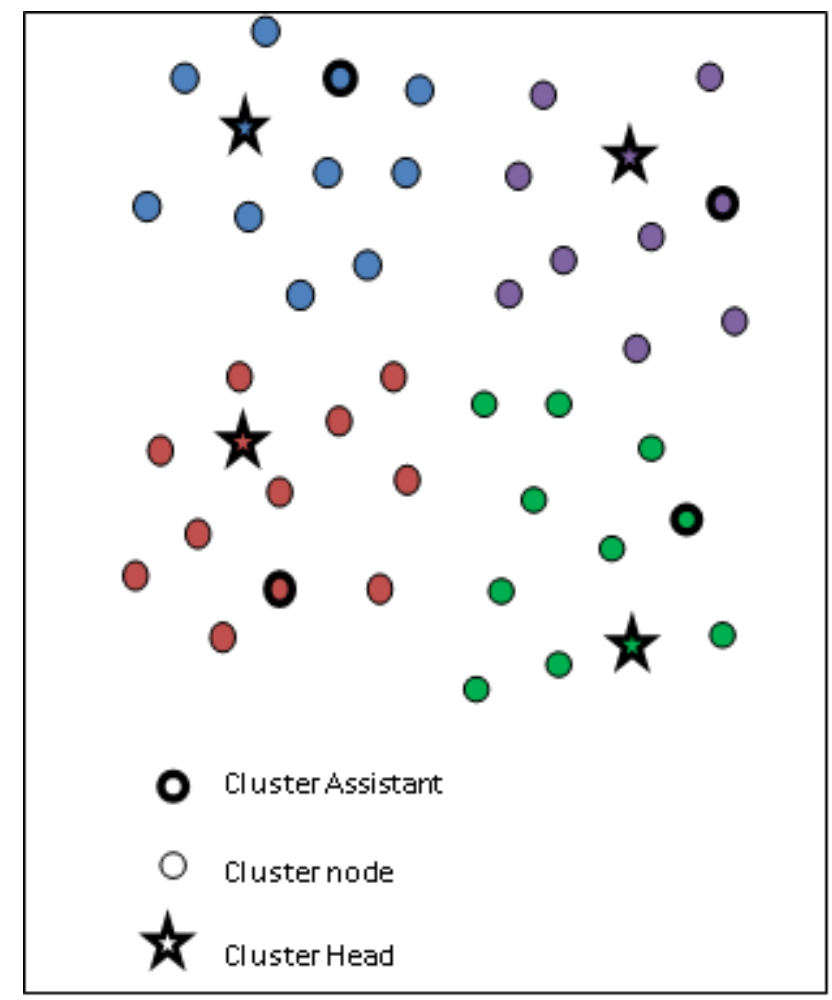

Fig. 4. Cluster Head with Cluster Assistant in each cluster

\section{Results and Discussions}

For simulation purpose of the proposed work, Network Simulator -2 (NS-2) tool is used under Linux platform. NS-2 is an open tool which supports both wired and wireless networks [5]. A wireless sensor network scenario of $200 * 200 \mathrm{~m}^{2}$ is considered with the initial energy of each sensor node as 3 Joules. The transmitting and receiving powers are 0.01 and 0.02 watts respectively. The output values are shown both in the text and graphical interface for various parameters. The proposed work is contrasted with the present LEACH and OEERP protocols.

Parameters such as energy consumption, PDR and lifetime are compared. The total energy consumption is the overall energy consumed by all sensor nodes during various process including sensing, transmitting and receiving. The total energy consumption is given by equation 5 .

$$
\text { Total Energy Consumption }=\sum_{N=1}^{n}\left[E_{N}(\text { initial })-E_{N}(\text { remaining })\right]
$$

Figure 5 shows the total energy consumption of the proposed work comparison with the existing LEACH and OEERP protocols. It reveals that the red line (proposed work) provides reduced energy consumption. 


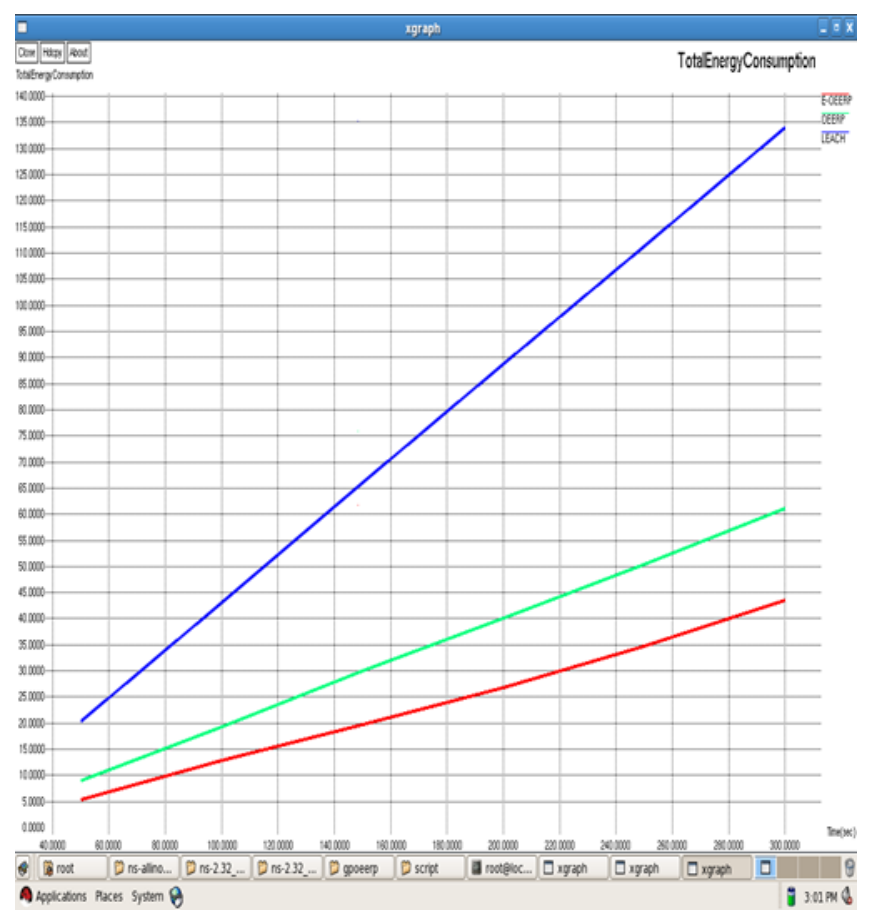

Fig. 5 Total Energy Consumption

Packet Delivery Ratio is defined as the number of packets delivered to the number of packets actually transmitted. It is given by equation 6

Packet Delivery Ratio=No of packets transmitted/No of packets transmitted

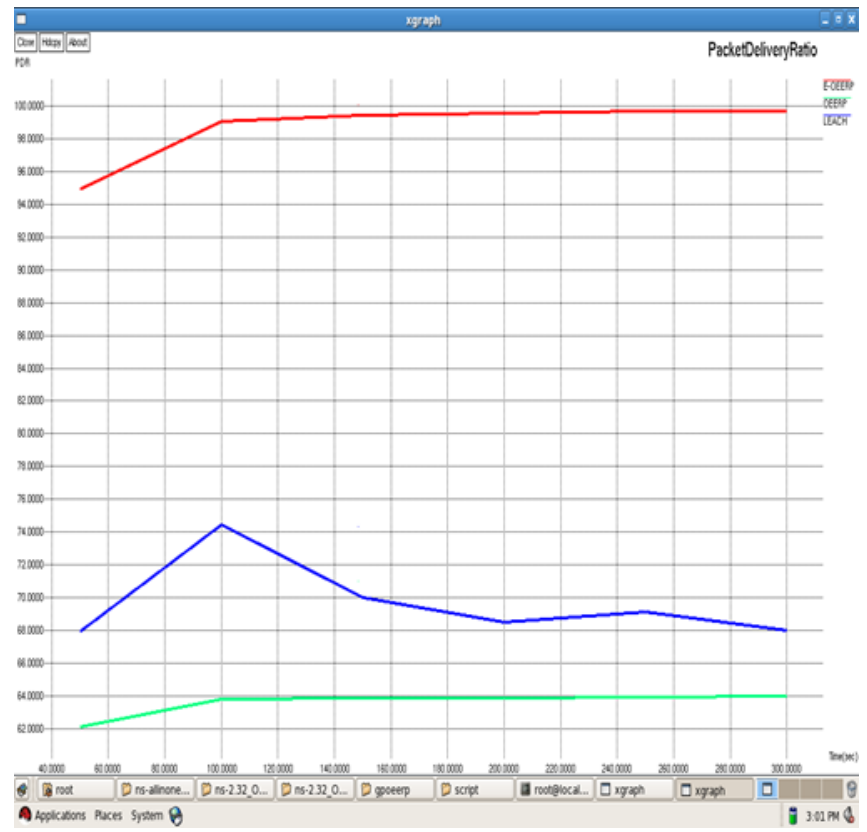

Fig. 6. Packet Delivery Ratio 
Figure 6 shows the PDR comparison of the proposed protocol with that of LEACH and OEERP protocols. It shows that the proposed protocol has high PDR compared with that of the existing protocols.

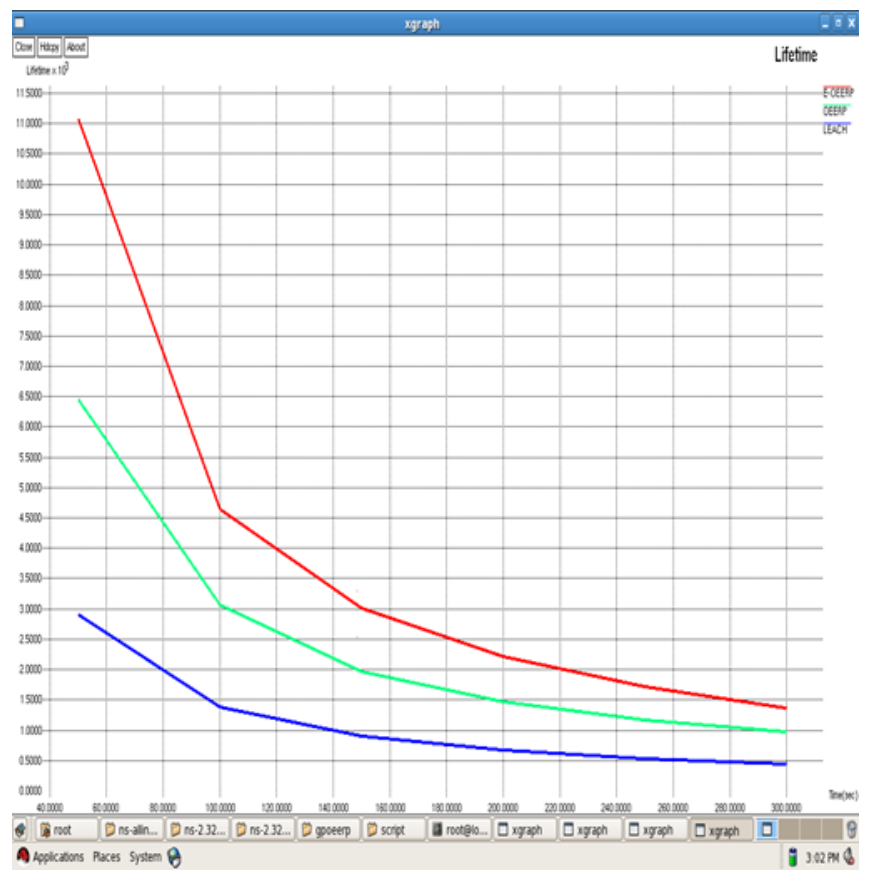

Fig. 7. Network Lifetime

Figure 7 provides the comparative graph for the proposed work with that of the existing protocols. Network Lifetime is a vital parameter in WSN which reveals the life-time of sensor nodes in the wireless network. Efficient use of battery amplify the lifecycle of sensor nodes which increases the lifecycle of the sensor network.

\section{Conclusion}

In this paper, Efficient Cluster Formation Protocol (ECFP) is proposed which provides efficient clustering formation. It avoids the formation of individual node (non-cluster node) during clustering as in the existing protocols. This can be achieved by using Particle Swarm Optimization algorithm for cluster structure and $\mathrm{CH}$ election by computing the fitness value for each particle. Prevention of individual node formation is necessary because of the reason that those nodes consume high energy in transmitting data to the BS. The proposed protocol achieves low energy consumption and high PDR compared to the existing protocols which is revealed from the simulation results.

\section{References}

[1] Sahu, E. B. Fernandez, Mihaela Cardei, and M. VanHilst, "A pattern for a sensor node", Procs. 17th Conf. on Pattern Languages of Programs (PLoP 2010).

[2] Jennifer Yick, Biswanath Mukherjee, Dipak Ghosal, Wireless sensor network survey, Computer Networks, Volume 52, Issue 12, 22 August 2008, Pages 2292-2330, ISSN.

[3] Wendi Rabiner Heinzelman, Anantha Chandrakasan, and Hari Balakrishnan, "Energy-Efficient Communication Protocol for Wireless Micro sensor Networks", Proceedings of the 33rd Hawaii International Conference on System Sciences - 2000. 
[4] K. Kishan Chand, P Vijaya Bharati and B. Seetha Ramanjaneyulu, "Optimized Energy Efficient Routing Protocol for Life-Time Improvement in Wireless Sensor Networks", IEEE-International Conference On Advances In Engineering, Science And Management (ICAESM -2012) March 30, 31, 2012.

[5] Nitin Mittal, Davinder Pal Singh, Amanjeet Panghal, R.S. Chauhan, "Improved Leach Communication Protocol for WSN", NCCI 2010 -National Conference on Computational Instrumentation, March 19,20,2010.

[6] C. Cirstea, "Energy efficient routing protocols for Wireless Sensor Networks: A survey," 2011 IEEE 17th International Symposium for Design and Technology in Electronic Packaging (SIITME), Timisoara, 2011, pp. 277-282.

[7] A. Wahid, S. Lee and D. Kim, "An energy-efficient routing protocol for UWSNs using physical distance and residual energy," OCEANS 2011 IEEE - Spain, Santander, 2011, pp. 1-6.

[8] M. Dhivya, A. K. Potnuru and M. Sundarambal, "Energy Efficient Routing Protocol for Wireless Sensor Networks," 2011 International Conference on Process Automation, Control and Computing, Coimbatore, 2011, pp. 1-5.

[9] M. Dhivya, A. K. Potnuru and M. Sundarambal, "Energy Efficient Routing Protocol for Wireless Sensor Networks," 2011 International Conference on Process Automation, Control and Computing, Coimbatore, 2011, pp. 1-5.

[10] Myung Kyun Kim and H. P. Ngo, "A reliable and energy efficient routing protocol in industrial wireless sensor networks," The 2011 International Conference on Advanced Technologies for Communications (ATC 2011), Da Nang, 2011, pp. 32-35.

[11] S. Preethi and B. Ramachandran, "Energy efficient routing protocols for mobile AdHoc networks," 2011 International Conference on Emerging Trends in Networks and Computer Communications (ETNCC), Udaipur, 2011, pp. 136-141. 